\title{
Brain ultrastructure: Putting the pieces together
}

Patrick C. Nahirney and Marie-Eve Tremblay

Division of Medical Sciences

Corresponding author:

University of Victoria, B.C., Canada

Patrick C. Nahirney

Division of Medical Sciences

University of Victoria

Victoria, B.C. Canada

Tel.: 250 853-3659

email: nahirney@uvic.ca

\section{Supplemental Data}

Supplemental figure:

1. High-resolution zoomable EM image of the mouse brain cortex showing the extent of astrocytes (shaded red) between neuronal processes and other glial cells. Astrocytes function to maintain the internal environment for neurons and glia. Two large pyramidal neurons are in the upper left and a capillary is in the center of the image. A typical microglia is in the lower left adjacent to a large dendrite. JPG, 11000 x 7616 pixels, $10 \mathrm{MB}$.

Supplemental videos:

1. EM tomography tilt series (+/- 25 degrees, 5 degree intervals) showing two spines (above, containing PSDs) synapsing with a glutamatergic presynaptic terminal filled with $\sim 40 \mathrm{~nm}$ diameter vesicles. MP4 video, $1 \mathrm{MB}$.

2. FIB-SEM z stack video of the mouse hippocampus showing the difference between typical and dark microglia. Both of these resident immune cells are in close apposition to a capillary. Imaging was performed at a $14 \mathrm{~nm}$ resolution in $\mathrm{x}, \mathrm{y}$, and $\mathrm{z}$ planes. MP4 video, $20 \mathrm{MB}$. 